ESJ Social Sciences

\title{
Influence du prix et de la communication dans l'industrie automobile sur la perception de l'image de marque: Cas du grand Agadir au Maroc
}

\author{
Amira Qanqom \\ Enseignant chercheur, FSJES Ait melloul, \\ Université Ibn Zohr, Agadir, Maroc
}

Doi:10.19044/esj.2021.v17n19p111

Submitted: 05 April 2021

Accepted: 18 May 2021

Published: 30 June 2021
Copyright 2021 Author(s)

Under Creative Commons BY-NC-ND

4.0 OPEN ACCESS

Cite As:

Qanqom A. (2021). Influence du prix et de la communication dans l'industrie automobile sur la perception de l'image de marque: Cas du grand Agadir au Maroc.

European Scientific Journal, ESJ, 17(19), 111. https://doi.org/10.19044/esj.2021.v17n19p111

\section{Resume}

Cet article tente de tester l'influence du prix et de la communication sur la perception de l'image de marque automobile. En effet, nous présentons une brève analyse du concept de l'image de marque, ainsi qu'une description du secteur de l'automobile dans notre pays et dans notre région. Enfin, nous exposons les résultats de l'étude quantitative réalisée, sur un échantillon de 385 personnes, composé de consommateurs et de non consommateurs de produits automobiles. Les résultats obtenus par la méthode des modèles d'équations structurelles montrent les liens entre les variables prix et communication avec la perception de l'image de marque automobile. En effet, nous avons pu prouver que la variable prix rentre dans le processus de construction de l'image de marque chez le consommateur. Contrairement à la communication dont la relation avec l'image de marque a été réfuté.

Mots clés : Image de marque, automobile, perception, prix, communication 


\title{
Influence of Price and communication on the automotive brand image perception: Greater Agadir case
}

\author{
Amira Qanqom \\ Enseignant chercheur, FSJES Ait melloul, \\ Université Ibn Zohr, Agadir, Maroc
}

\begin{abstract}
The aim of the article is to test the influence of price and communication on the perception of automotive brand image. Indeed, we present a brief analysis of the brand image concept, as well as a description of the automotive field in the country, and also in the region. Finally, we report the results of the survey, based on a sample of 385 persons of automotive products' consumers and non-consumers. Using the structural modeling technique, the results reveal the effect of price and communication on the automotive brand image perception. Indeed, we have proved that the variable price is part of the process of developing a brand image for the consumer. Unlike communication, the relation with brand image hasn't been proven in our case of study.
\end{abstract}

Keywords: Brand image, automotive, perception, price, communication

\section{Introduction}

Il est incontestable que les marques représentent un élément incontournable de notre société de consommation. Elles sont partout autour de nous, et font partie de notre quotidien, au travail, à la maison, dans la rue, impossible d'y échapper. Nous entretenons une relation avec les marques qui est de plus en plus forte. Ainsi, nous ne mangeons pas un yaourt ou une gaufrette, mais un Danone et un Tonic; pour acheter une lessive on demande à l'épicier du coin un Tide, car c'est le synonyme de la propreté. Force est de constater qu'elles se sont littéralement immiscées dans nos vies, nous pouvons être sensibles, fidèles ou encore attachés à une marque. En effet, la question qui se pose est : Pourquoi choisit-on une marque au lieu d'une autre ? Si vous avez la réponse, sachez que vous ferez l'objet de convoitise pour les stratèges du marketing. Avant de tenter de répondre à une question aussi complexe, intéressons nous à l'image de marque.

L'image de marque, selon Aaker (1994), et Aghekyan-simonien et al. (2012), est un construit complexe et intuitif. Cependant, même si elle a été reconnue comme un concept pertinent dans le marketing, il y a une absence de consensus autour de sa mesure et de sa modélisation Salciuviene et al. (2007). 
Rappelons que Keller (1993) définit l'image de marque en tant que perceptions d'un ensemble d'associations relatives à la mémoire des consommateurs. En outre, Keller $(1993,2009)$ indique que l'image de marque est définie non seulement par ses associations, mais également par sa qualité d'association (positive ou négative) dans l'esprit du consommateur, l'intensité ou la force d'association (forte, faible, inexistante), et de l'unicité (association unique, non partagé avec d'autres marques). L'image de marque est reflétée par un ensemble d'images perçues par les clients Dobni et Zinkhan (1990). Dans une autre optique, la perception d'image de marque est un ensemble de pensées, sensations et attentes relatives à une marque donnée, Huang (2010). Malgré l'apport managérial et théorique des recherches existantes, il subsiste une carence au niveau des recherches empiriques spécifiques, ayant pour but de comprendre la construction de la perception des consommateurs des marques, Schmitt (2012). En effet, Cian (2011) stipule qu'au niveau de la formulation théorique d'image de marque, il est logique de penser que ses techniques de mesure ne sont pas encore normalisées. En raison du nombre élevé d'éléments qui déterminent l'image de marque, sa mesure demeure difficile à réaliser. Cela est principalement dû au fait que les perceptions qui composent l'image d'une marque sont multiples et peuvent se référer à des aspects tangibles et intangibles qui, en outre, dépendent de "l'évaluation interne » de chaque individu, Aaker (1994). Pour mener notre étude, nous avons chois le secteur automobile comme champs d'application.

\section{Le secteur automobile comme champs d'étude}

Notre choix s'est porté sur le secteur de l'automobile en raison de la pertinence de ce champ d'étude et de sa richesse. En effet, selon Morisse, (2004), ce secteur est souvent sujet aux théories du management et du marketing, Soulez (2005). De plus, la marque y détient une place importante et entraine un impact sur la construction du pouvoir de marché des constructeurs, Morisse (2004). D‘un autre côté, selon le même auteur, qu'il soit question du marché de véhicules neufs, ou d'occasion, l'image de marque et la réputation constituent des antécédents directs dans la décision d'achat.

En effet, Voelckner et Satler (2005) ont réalisé des études, qui ont révélé 1'importance de l'image de marque, comme étant un facteur clé de la réussite d'une entreprise. Ces déductions affectent tout particulièrement le

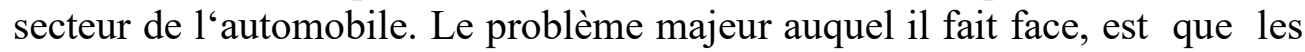
marques automobiles offrent des performances et des caractéristiques assez proches, et perdent ainsi leur capacité de se différencier. Ainsi Les constructeurs automobiles considèrent l'image de marque comme facteur fondamental de différenciation.

Depuis 2010, nous avons commencé à remarquer l'évolution de l'image de plusieurs constructeurs; nouveaux logos, nouveaux efforts 
publicitaires, nouvelles ambitions. Tout cela est fait dans le but de créer une perception positive de la part du public. Ainsi, en moins de deux ans, une véritable course au «relooking» visuelle a été entamée: Audi, Citroën, $B M W \ldots$ tout le monde s'y est mis. Bref, il est clair que l'industrie automobile l'a bien compris, il faut travailler son image pour vendre.

En ce qui concerne le Maroc, l'industrie automobile a connu un essor considérable, nous avons aujourd'hui une capacité de fabrication de 650.000 véhicules par an, et faisons partie des grands fabricants de l'automobile dans le monde, avec l'objectif d'atteindre 1 million de véhicules. En ce qui concerne les ventes, les statistiques de l'AVIAM (Association Association des importateurs de véhicules automobiles) démontrent qu'elles sont en constante progression. Cela vient récompenser les efforts fournis par les marques automobiles dans le but de véhiculer une image positive auprès du public.

D'un autre coté, Le Maroc compte aujourd'hui plus de 33 millions d'habitants ${ }^{1}$, et nous somme connus pour être un peuple dont les habitudes et les comportements sont assez diversifiés selon les régions. Pour ces raisons, et pour que nous puissions mener une étude en bonne et du forme, nous allons limiter notre étude sur le grand Agadir.

Suite à la croissance considérable que le secteur automobile connait dans notre pays, la région du Souss Massa en général, et le grand Agadir en particulier, représente un relais intéressant, selon le président de l'Association Marocaine pour l'industrie et le Commerce de l'Automobile (M. Hakim Abelmoumen).

Enfin, selon les statistiques révélées par l'Association des importateurs de véhicules automobiles, le grand Agadir est en quatrième position des ventes de voitures privées au Maroc, au titre de l'année 2016. Elle a été classée derrière les villes de Marrakech, Rabat et Casablanca.

Il serait pertinent de mener notre étude au niveau national (Maroc), cependant il est difficile de toucher un échantillon représentatif, du fait que la taille de la population est trop importante. De ce fait, notre étude portera sur la population du grand Agadir.

\section{Typologie de l'image de marque}

Avant d'entamer l'étude réalisée, nous présentons la typologie de l'image de marque basée principalement sur les associations à la marque, en d'autre termes, ce sont des nœuds qui y sont liés Korchia (2001). Les associations peuvent être aussi définies comme les nœuds informationnels liés au nœud de la marque dans la mémoire et contiennent le sens de la marque par le consommateur, Keller (1993).

\footnotetext{
${ }^{1}$ Selon le haut commissariat au plan : https://www.hcp.ma/ (Consulté en avril 2018)
} 
La typologie ci-dessous, a été le résultat d'une étude qualitative que nous avons préalablement mené, moyennant des entretiens semi directifs et suivant un guide d'entretiens. Ce dernier a été élaboré en se basant sur le travail de Korchia (2001) et également sur la procédure d'élicitation libre ${ }^{2}$.'échantillon était composé de 23 personnes, ce nombre a été déterminé suivant le principe de saturation. Agés entre 23 et 58 ans, ayant le permis de conduire et possédant ou désirant posséder une voiture. Toutes ces personnes ont un intérêt pour l'automobile, connaissent les différentes marques qui existent sur le marché, et sont donc des consommateurs ou futurs consommateurs. Il est à noter que 5 d'entre ces interviewés sont des professionnelles de l'automobile.

Figure 1: typologie de l'image de marque retenue pur l'étude

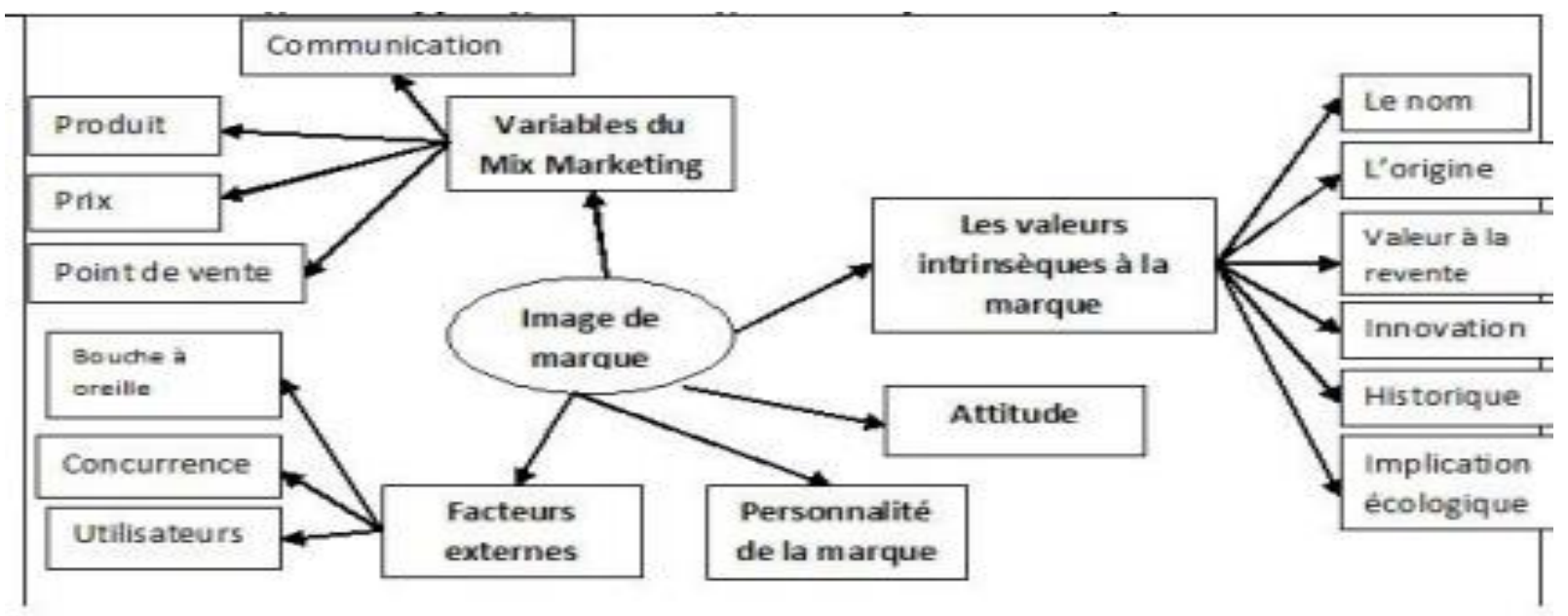

Source: Qanqom (2018)

A présent intéressons nous à l'influence du prix et de la communication sur la conception de l'image de marque automobile.

\section{Hypothèses de l'étude}

Pour la catégorie de prix, la littérature en Marketing le considère comme un facteur qui peut influencer l'image de marque. En effet, du point de vue du consommateur, le prix constitue un indicateur de la qualité, Erickson et Johansson (1985); Rao et Monroe (1989) ; Dodds, Monroe et Grewal (1991) ; Kim et Hyun (2011). Selon les travaux de ces auteurs, il aurait une

${ }^{2}$ La méthode d'élicitation libre est définit par (Olson et muderrisoglu, 1979), comme « une procédure dans laquelle les répondants sont entièrement libres de dire tout ce qui leur vient à l'esprit lorsqu'on leur présente un stimulus de départ ». 
relation directe avec la perception du consommateur de la marque. Sur la base de ces points, nous pouvons émettre l'hypothèse suivante: H1 : le prix influence la construction d'image de marque par le consommateur.

Concernant la communication, l'impact des effets de la publicité, les événements promotionnels, la vente directes, sur la perception de la marque ont été analysées dans plusieurs recherches, Simon \& Sullivan (1993); Yoo \& Donthu, 2001; Yoo et al. (1993). Les événements promotionnels avec des objectifs à long terme pourraient construire une image de marque positive en offrant une expérience réelle du produit, ce qui aide à créer des associations «fortes, favorables et uniques » Keller (2009). Dans le marketing industriel, des activités promotionnelles telles que des brochures et les sites Web sont fréquemment mentionnés comme des outils influençant la valeur que le consommateur donne à la marque, Sharma, Krishnan, et Grewal (2001); van Riel et al. (2005). Sur la base de ces éléments, la $2^{\text {ème }}$ hypothèse de recherche est la suivante : $\mathbf{H 2}$ : la communication influence la construction d'image de marque par le consommateur.

\section{Opérationnalisation des variables}

Nous développons ci-après l'ensemble des items utilisés pour collecter les données. Tous ces items sont issus d'échelles fiables, de sources fiables, ou encore de propos tenus par les répondants lors de phase qualitative. Pour ce travail, nous allons présenter les variables prix et communication.

\section{Mesure de la variable « Prix »}

Les voitures ne font pas l'objet d'achats fréquents, c'est en grande partie dû à l'importance primordiale du prix lors de l'achat. Il sera donc question de mesurer l'importance du prix par rapport à la perception de l'image de marque. À cet égard, il existe une échelle développée par Sproles et Sproles (1990), qui se compose de trois items : achat des produits en solde, achat des produits ayant le prix le plus bas, et le rapport qualité prix ${ }^{3}$.

Nous nous referons donc à cette échelle, en complément des résultats de l'étude qualitative. Cette variable est automatiquement associée à la qualité, de ce fait nous l'avons mesuré par le rapport qualité/prix. A coté de cela il existe, le prix de la voiture et les coûts engendrés (entretien, vignette, assurance).

\section{Mesure de la variable « Communication »}

Dans la littérature, ce concept est très large. En ce qui concerne ces échelles de mesures, elles sont plus relatives à l'efficacité de la communication

\footnotetext{
${ }^{3}$ Nous n'avons pas réussis à trouver« alpha de Cronbach » relatif à cette échelle dans cet article de référence, Cependant, sa source est fiable et nous accepterons donc cette échelle (Journal of Consumer Affairs).
} 
par exemple, ou son influence sur le consommateur. Beaucoup d'auteurs se sont intéressé à cette mesure, Aaker et al (1986) ; Zinkhan et Fornell, (1985), qui se résume à établir une liste d'adjectifs pour pouvoir identifier les dimensions sous-jacentes de la réaction du consommateur aux actions de communications des marques et en particulier la publicité.

Dans le cas de notre étude, nous avons réussi à mettre la main sur les principales techniques de communications considérées par les répondants dans le domaine de l'automobile : publicité, courriers, e-mails, SMS, actions de parrainage et presse spécialisée. Il sera donc question de mesurer le degré d'influence de ces outils, par rapport à la construction de l'image de marque dans l'esprit du consommateur. Cela est fait moyennant une échelle de Likert en 5 échelons.

Nous avons également utilisé diverses mesures de plus, qui nous permettrons de contrôler des sources de variances. En effet, il nous parait utile de connaitre si les répondants sont en possessions ou non du produit dont ont sollicite le jugement. Elles nous permettent également de stratifier notre échantillon.

Le tableau suivant résume les items correspondants à chaque variable.

Tableau 1 : Liste des items correspondant à chaque variable

\begin{tabular}{|l|l|}
\hline $\begin{array}{l}\text { Les variables } \\
\text { indépendantes }\end{array}$ & Les items correspondants \\
\hline Prix: 3 & $\begin{array}{l}\text { le rapport qualité/prix. le prix de la voiture et les coûts } \\
\text { engendrés (entretien, vignette, assurance), }\end{array}$ \\
\hline Communication: 4 & $\begin{array}{l}\text { La publicité, les actions de communication de la marque en } \\
\text { général, à savoir (les courriers, e-mails, SMS), le parrainage } \\
\text { et la presse spécialisée. }\end{array}$ \\
\hline
\end{tabular}

\section{Validité du questionnaire}

Une fois que le questionnaire fut établi ; nous avons réalisé ce que l'on appelle la «validité faciale du questionnaire ». Ceci revient à valider son contenu, de façon à ce que les items traduisent effectivement les variables mesurées, dans le contexte du marché marocain. A ce titre, nous l'avons soumis à un directeur de concession automobile, ayant une longue expérience dans la vente des voitures, et une grande connaissance du consommateur marocain de ce genre de produits. Suite à cet examen, il nous a été conseillé de reformuler certaines questions, afin qu'elles puissent être mieux et facilement assimilées par les répondants. Mais, globalement, le questionnaire a été validé en totalité.

\section{Définition de l'échantillon}

A ce stade, nous précisons quelle sera la population étudiée, son unité d'analyse, la stratégie pour constituer un échantillon et la taille de cet échantillon. Dans la majorité des cas, la population choisie par le chercheur 
sera trop large pour que ce dernier puisse envisager de recueillir des données auprès de tous les éléments qui la composent. Selon (Evrard et al, 2000), le choix de la population de base se fait, généralement, à partir de deux grandes catégories de méthodes de sondage ou d'échantillonnage. La différence entre les deux méthodes tient à la nature de la population étudiée. On distingue : Les méthodes aléatoires ou probabilistes et les méthodes non probabilistes ou non aléatoires.

Pour des raisons liées au mode d'administration du questionnaire, notre échantillon s'inscrit dans la catégorie des échantillons non aléatoires ou non probabilistes. Nous avons utilisé dans notre recherche, la méthode du choix raisonné. Le recours à cette méthode donne de bons résultats lorsqu'on cherche à tester des propositions théoriques. Les échantillons sélectionnés par cette méthode sont fréquemment rencontrés dans les recherches en sciences sociales. C'est une méthode rapide, moins coûteuse et permet de choisir de manière très précise les éléments de l'échantillon, chose qui rend facile le respect des critères fixés par notre recherche. Par conséquent, nous avons déterminé les points suivants relatifs à l'échantillonnage :

\section{Population de référence}

La population de cette étude est composée de plusieurs strates. La première concerne les personnes possédant une voiture et la deuxième concerne ceux qui n'en possèdent pas. En effet, il nous a parut claire à travers l'étude qualitative préalablement réalisée, que les personnes peuvent avoir une vision très nette de l'image de marque automobile, sans pour autant en posséder une. Il faut aussi préciser que pour des raisons de commodité, nous allons nous concentrer sur la population du grand Agadir.

\section{Méthode d'échantillonnage}

Pour respecter la logique que nous venons d'exposer, nous allons donc utiliser la méthode d'échantillonnage stratifié non proportionnel.

L'administration du questionnaire à notre échantillon se fera dans différents endroits pour pouvoir toucher toutes les strates (personnes motorisées et non motorisées).

Pour les deux strates, nous allons cibler les grandes surfaces, les administrations, les institutions de formation ainsi que toute autre lieu qui pourrait présenter une opportunité pour collecter les informations désirées.

\section{Taille de l'échantillon}

Selon les lois de la statistique, un échantillon est considéré comme statistiquement significatif dans la mesure où il compte au moins 30 répondants choisis de façon aléatoire. Cependant, plus la taille de l'échantillon 
est grande, plus l'analyse sera précise. Mais encore, l'analyse ne sera pas 2 fois plus précise, si notre échantillon est 2 fois plus grand.

Ainsi, la détermination de la taille de l'échantillon est une étape cruciale dans une étude. Trois facteurs déterminent particulièrement la taille de l'échantillon:

- La prévalence estimative d'une variable étudiée : dans notre cas, nous prendrons la variable concernant la question 12 de notre questionnaire (la proportion estimative des personnes de la population qui pourraient être influencées par l'image de marque pour changer leur comportement d'achat.),

- Le niveau de confiance visé,

- La marge d'erreur acceptable.

Pour notre cas d'échantillonnage, nous pouvons calculer la taille d'échantillon en appliquant la formule suivante.

$$
n=\frac{Z^{2} \alpha / 2 \times p \times(1-p)}{e^{2}}
$$

- $\mathrm{n}$ : taille de l'échantillon attendu.

- $Z \alpha / 2$ : niveau de confiance. Généralement, on prend un niveau $Z \alpha / 2$ de 1,96 qui lié à un taux de confiance de $95 \%$,

- $\mathrm{p}$ : proportion estimative de la population présentant la caractéristique étudiée dans l'étude. Lorsque cette proportion est ignorée, une préétude peut être réalisée ou sinon un p égal à 0,5 sera retenue.

- e : marge d'erreur (traditionnellement fixée à 5\%).

Une application numérique nous permet d'estimer la taille globale de notre échantillon.

$$
\mathrm{n}=\frac{1.96^{2} \times 0,5(1-0,5)}{0,05^{2}} \quad \mathrm{n}=\frac{0,9604}{0,0025}
$$

$\mathrm{n}=384,16$ E 385 personnes

Suite donc à nos calculs, nous allons choisir un échantillon global de 385 personnes qui seront réparties comme suit selon les strates expliqués dans les lignes précédentes : 
Tableau 2 : Répartition des strates de l'échantillon

\begin{tabular}{|c|c|c|}
\hline Strates & \multicolumn{2}{|c|}{ Nature des informations souhaitées } \\
\hline Personnes motorisées & $\begin{array}{l}\text { Perception de l'image } \\
\text { de la marque de voiture } \\
\text { qu'elles possèdent. }\end{array}$ & $\begin{array}{l}\text { Influence } \\
\text { de leur } \\
\text { perception }\end{array}$ \\
\hline Personnes non motorisées & $\begin{array}{l}\text { Perception de l'image } \\
\text { de marque automobile } \\
\text { en général. }\end{array}$ & $\begin{array}{l}\text { sur le } \\
\text { comporte } \\
\text { ment } \\
\text { d'achat }\end{array}$ \\
\hline
\end{tabular}

Source : Conception personnel

Cet échantillon n'est pas constitué de professionnels de l'automobile, car la nature des informations que nous ciblons se situe au niveau des consommateurs non experts. Toutes les personnes sont âgées d'au moins 18ans, et disposent obligatoirement d'un permis de conduire. Enfin, ce sont des consommateurs, ou futurs consommateurs d'automobiles.

\section{Analyse et résultats}

L'objectif de ce point est de présenter l'analyse exploratoire des réponses des interviewés à notre questionnaire (concernant les variables retenues pour cette étude). Nous allons alors vérifier la validité et la fiabilité des items/construits à travers le logiciel SPSS. Cette tâche sera effectuée principalement par le biais de l'analyse factorielle (ACP) et de l'analyse de fiabilité qui nous permettront d'aboutir à l'épuration de notre modèle de recherche. Commençons par présenter les résultats pour le prix en vérifiant son unidimensionnalité et sa fiabilité.

\section{Variable Prix}

Tableau 3 : Résultats de l'ACP sur les échelles de prix

\begin{tabular}{|c|c|c|c|c|c|c|}
\hline Items & $\begin{array}{l}\text { Test de } \\
\text { caractère } \\
\text { factorisable }\end{array}$ & Communauté & Facteur & $\begin{array}{l}\text { Valeur } \\
\text { propre }\end{array}$ & $\begin{array}{l}\text { \% de la } \\
\text { variance }\end{array}$ & $\begin{array}{l}\text { Contributions } \\
\text { factorielles }\end{array}$ \\
\hline Prix 1 voit & \multirow{3}{*}{$\begin{array}{l}\text { KMO }=0,648 \\
\text { Test de } \\
\text { Bartlett } \\
\text { Sig }=, 000\end{array}$} & ,610 & \multirow[t]{3}{*}{1} & 1,740 & 57,994 & ,781 \\
\hline Prix 2 qualité & & ,593 & &, 671 & &, 770 \\
\hline Prix 3 coûts & &, 537 & &, 589 & &, 733 \\
\hline
\end{tabular}

Source : Conception personnelle à partir de SPSS

$\mathrm{Au}$ vu de ces résultats, les indices de communauté sont supérieurs et la représentation factorielle est satisfaisante, avec un pourcentage de variance totale expliquée de 58\%. L'unidimensionnalité des items de prix est donc confirmée. 
Tableau 4: Analyse de la fiabilité des items du prix

\begin{tabular}{|l|l|l|l|l|}
\hline Items & Moyenne & Ecart-type & $\begin{array}{l}\text { Corrélation } \\
\text { entre les items } \\
\text { et le score de } \\
\text { l'échelle }\end{array}$ & $\begin{array}{l}\text { Coefficient } \\
\text { Alpha si l'item } \\
\text { est éliminé }\end{array}$ \\
\hline Prix 1 voit & 4,05 &, 997 &, 410 & - \\
\hline Prix 2 qualité & 4,32 &, 892 &, 358 & - \\
\hline Prix 3 coûts & 3,75 & 1,124 &, 341 & - \\
\hline
\end{tabular}

Source : Conception personnelle à partir de SPSS

Le calcul de l'indice de fiabilité Alpha de Cronbach nous donne : 0,637 .

$\mathrm{Au}$ vu de ces résultats, les indices de communauté sont supérieurs et la représentation factorielle est satisfaisante, avec un pourcentage de variance totale expliquée de 58\%. L'unidimensionnalité des items de prix est donc confirmée.

\section{Variable Communication}

Tableau 5: Résultats de l'ACP sur les échelles de communication

\begin{tabular}{|c|c|c|c|c|c|c|}
\hline Items & $\begin{array}{l}\text { Test de } \\
\text { caractère } \\
\text { factorisable }\end{array}$ & Communauté & Facteur & $\begin{array}{l}\text { Valeur } \\
\text { propre }\end{array}$ & $\begin{array}{l}\text { \% de la } \\
\text { variance }\end{array}$ & $\begin{array}{l}\text { Contributions } \\
\text { factorielles }\end{array}$ \\
\hline Comm 1 pub & \multirow{4}{*}{$\begin{array}{l}\mathrm{KMO}=, 702 \\
\text { Test de } \\
\text { Bartlett } \\
\text { Sig=,000 }\end{array}$} & ,522 & \multirow[t]{4}{*}{1} & 1,967 & $49,165 \%$ & ,723 \\
\hline $\begin{array}{l}\text { Comm } 2 \\
\text { courrier }\end{array}$ & & ,353 & & ,803 & & ,594 \\
\hline $\begin{array}{l}\text { Comm } 3 \\
\text { parrain }\end{array}$ & & ,524 & & ,679 & & ,724 \\
\hline $\begin{array}{l}\text { Comm } 4 \\
\text { presse }\end{array}$ & & ,567 & & ,551 & & ,753 \\
\hline
\end{tabular}

Source : Conception personnelle à partir de SPSS

Les résultats de l'ACP montrent des seuils moyens pour les indices de communautés et pour les contributions factorielles mais elles démontrent néanmoins l'unidimensionnalité des items de la communication.

Tableau 6: Analyse de la fiabilité des items de communication

\begin{tabular}{|l|l|l|l|c|}
\hline Items & Moyenne & Ecart-type & $\begin{array}{l}\text { Corrélation } \\
\text { entre les items et } \\
\text { le score de } \\
\text { l'échelle }\end{array}$ & $\begin{array}{l}\text { Coefficient } \\
\text { Alpha si l'item } \\
\text { est éliminé }\end{array}$ \\
\hline Comm 1 pub & 2,98 & 1,293 & 0,289 & 0,568 \\
\hline Comm 2 courrier & 2,52 & 2,025 & 0,316 & 0,654 \\
\hline Comm 3 parrain & 2,67 & 1,275 & 0,395 & 0,573 \\
\hline Comm 4 presse & 2,91 & 1,200 & 0,419 & 0,549 \\
\hline
\end{tabular}

Source : Conception personnelle à partir de SPSS 
Le calcul de l'indice de fiabilité Alpha de Cronbach nous donne : 0,652 .

Les résultats de l'ACP montrent des seuils moyens pour les indices de communautés et pour les contributions factorielles mais elles démontrent néanmoins l'unidimensionnalité des items de la communication.

\section{Validation des échelles}

Cette étape a consisté à analyser la contribution factorielle (loading) et le t-student de chaque item, la variation restituée par chaque dimension (AVE), la fiabilité composée (CR) et l'Alpha de Cronbach $(\alpha)$. Les résultats obtenus sont présentés dans les tableaux suivants :

Tableau 7 : Résultats de la validation des variables du Prix

\begin{tabular}{|c|l|c|}
\hline \multicolumn{2}{|c|}{ Paramètres } & Modèle 1 \\
\hline $\begin{array}{c}\text { Contributions factorielles (t- } \\
\text { student) }\end{array}$ & Prix 1 (prix voit) & $0.751(17.982)$ \\
\cline { 2 - 3 } & Prix 2 (quali prix) & $0.722(14.789)$ \\
\cline { 2 - 3 } & Prix 3 (coût) & $0.740(17.494)$ \\
\hline Indicateurs & \multicolumn{1}{|c|}{ Modèle1 } & \\
\hline $\boldsymbol{A V E}$ & Prix & 0.5441 \\
\hline $\boldsymbol{C R}$ & Prix & 0.7816 \\
\hline Alpha de Cronbach & Prix & 0.5809 \\
\hline
\end{tabular}

Source : Conception personnelle à partir de SmartPLS

Tableau 8: Résultats de la validation des variables de Communication

\begin{tabular}{|c|c|c|}
\hline \multicolumn{2}{|c|}{ Paramètres } & Modèle 1 \\
\hline \multirow{3}{*}{$\begin{array}{c}\text { Contributions } \\
\text { factorielles (t-student) }\end{array}$} & Communicat 1 (pub) & $0.638(12.129)$ \\
\cline { 2 - 3 } & $\begin{array}{c}\text { Communicat 2 (courrier, } \\
\text { email, sms) }\end{array}$ & $0.824(33.772)$ \\
\cline { 2 - 3 } & Communicat 3(parrainage) & $0.821(28.91)$ \\
\cline { 2 - 3 } & Communicat 4 (presse) & $0.750(20.331)$ \\
\hline Indicateurs & Modèle1 & 0.5805 \\
\hline $\boldsymbol{A V E}$ & Communication & 0.8457 \\
\hline $\boldsymbol{C R}$ & Communication & 0.7551 \\
\hline Alpha de Cronbach & Communication & \\
\hline
\end{tabular}

Source : Conception personnelle à partir de SmartPLS

Les indices de fiabilité de l'ensemble des échelles de mesure montrent que ces construits possèdent une cohérence interne élevée (CR et Alpha dépassant 0,7 ). En définitive, l'analyse factorielle confirmatoire nous a permis de valider et d'améliorer la représentativité des construits latents par leurs indicateurs réflexifs. Ainsi, elle a confirmé le caractère unidimensionnel des facteurs identifiés dans la première phase d'analyse. Par ailleurs, elle a permis de mieux «épurer» les échelles de mesure.

De façon générale, rappelons qu'un bloc de construit est dit réflexif, si l'on considère que les variables manifestes reflètent la variable latente, 
qu'elles en sont une conséquence. Un construit est dit formatif, si la variable latente est représentée par l'ensemble des variables manifestes, qu'elle en est une conséquence. Le test de nos deux hypothèses à travers PLS nous donne les résultats suivants :

Tableau 9 :Test des hypothèses en PLS

\begin{tabular}{|c|c|c|c|c|c|c|c|}
\hline \multicolumn{2}{|c|}{ Hypothèses } & $\begin{array}{c}\text { Original } \\
\text { Sample } \\
(\text { O) }\end{array}$ & $\begin{array}{c}\text { Sample } \\
\text { Mean } \\
(\mathbf{M})\end{array}$ & $\begin{array}{c}\text { Standard } \\
\text { Error } \\
\text { (STERR) }\end{array}$ & $\begin{array}{c}\text { Statistics } \\
\text { (|O/STE } \\
\text { RR/) }\end{array}$ & $\begin{array}{c}\text { Intervalle de } \\
\text { confiance } \\
\text { valeurs de } \mathbf{p}^{\mathbf{4}}\end{array}$ & \\
\hline $\begin{array}{c}\text { H } \\
1\end{array}$ & $\begin{array}{c}\text { Prix -> Image de } \\
\text { marque }\end{array}$ & 0.0941 & 0.0873 & 0,0577 & 1.6406 & 0.0467 & Sign \\
\hline $\begin{array}{c}\text { H } \\
2\end{array}$ & $\begin{array}{c}\text { Communication -> } \\
\text { Image de marque }\end{array}$ & 0.0275 & 0.0335 & 0,0448 & 0,6147 & 0.4605 & Pas sign \\
\hline
\end{tabular}

Source : Conception personnelle à partir de SmartPLS

Remarquons que pour des tests bilatéraux (en two-tail) et avec des degrés de liberté de 999 (puisque nous avons utilisé 1000 échantillons/samples dans le ré échantillonnage) nous avons obtenus des intervalles de confiance significatifs. A cet égard, nous rappelons également que le construit est dit statistiquement significatif au seuil de $1 \%, 5 \%$ et $10 \%$ si et seulement si son T de Student est supérieur à la valeur absolue de 2,57, 1,96 et 1,64 respectivement. $\mathrm{Au}$ dessous de ces seuils, la significativité des hypothèses semble être réduite (faible lien entre les variables supposées être explicatives et expliquées).

Par conséquent, Le prix influence la perception d'image de marque avec un test de student significatif $(t=1,64)$ mais l'ampleur de cet effet est réduite (original sample $=0,09$ ). Le prix est un facteur explicatif de l'image de marque.

Le tableau suivant synthétise nos résultats :

\begin{tabular}{|l|l|l|l|}
\hline Prix & $\begin{array}{l}\text { Concept unidimensionnel } \\
\text { mesuré par des items liés au } \\
\text { prix des voiture, le rapport } \\
\text { qualité prix et les coûts } \\
\text { engendrés. }\end{array}$ & $\begin{array}{l}\text { Le lien est } \\
\text { statistiquement } \\
\text { significatif. }\end{array}$ & $\begin{array}{l}\text { Cela appuie notre } \\
\text { hypothèse concernant le } \\
\text { prix et prouve que ce } \\
\text { dernier détient une } \\
\text { importante place pour la } \\
\text { construction de l'image de } \\
\text { marque automobile. }\end{array}$ \\
\hline Communication & $\begin{array}{l}\text { Concept unidimensionnel } \\
\text { mesuré par des items liés à la } \\
\text { publicité, les courriers, le } \\
\text { parrainage et presse } \\
\text { spécialisée. }\end{array}$ & $\begin{array}{l}\text { Le lien n'est pas } \\
\text { statistiquement } \\
\text { significatif. }\end{array}$ & $\begin{array}{l}\text { Les supports de } \\
\text { communication ne } \\
\text { représentent pas pour le } \\
\text { consommateur un moyen } \\
\text { de construire un image de } \\
\text { marque automobile. }\end{array}$ \\
\hline
\end{tabular}

Conception personnelle

${ }^{4}$ Les valeurs de p peuvent être calculées sur Excel directement en utilisant la formule suivante:

= DISTT(t-value; degrees.of.freedom-1;2). 


\section{Effet du prix sur la formation de l'image de marque}

Le prix est représenté dans notre étude par la gamme de prix des voitures, les coûts engendrés et le rapport qualité prix. A travers ces éléments, le consommateur Gadiri peut construire une image concernant les marques automobiles. Ce résultat est appuyé par celui de (Ratier, 2006), qui a également prouvé le rôle important du prix par rapport à la formation de l'image de marque, qui occupe pour le cas de son étude, la place de premier support de perception. Par conséquent, contrairement à un consommateur français qui se construit une image de marque automobile principalement à partir de son prix. Ce dernier se trouve en troisième place en matière de construction d'image de marque dans notre contexte d'étude.

\section{Conclusion}

Pour conclure, Le rôle de la communication par rapport à la formation de l'image de marque automobile, pour les consommateurs du grand Agadir a été infirmé. Par conséquent nous avons rejeté l'hypothèse selon laquelle la communication puisse avoir une influence sur la conception de l'image de marque. Nous avons donc constaté que les consommateurs ne se fient pas aux publicités en particulier, mais aussi aux autres actions de communication qu'une marque automobile peut faire. Dans la littérature concernant la publicité par exemple, on démontre qu'elle a un rôle de persuasion, qui peut être un processus cognitif ou affectif Holbrook et Batra (1987). En outre, selon Batra et al (1995), l'efficacité de la publicité peut être évaluée à partir de son effet sur les ventes, à court terme. Elle exerce également un impact plus important sur la formation de l'image du produit et l'image de marque Aaker et Carman, (1982). Enfin, selon Ratier (2006), qui a mené une étude assez similaire en France, il a affirmé le rôle de la publicité comme support de perception de l'image de marque. Dans notre cas, cette confirmation n'est pas applicable, du fait que les résultats de l'enquête menée attestent du contraire.

Par ailleurs, pour le choix de leurs voitures, les consommateurs marocains prennent en considération en premier lieu la marque, suivie de la motorisation et ensuite le prix ${ }^{5}$. Tout comme c'est un critère de choix pour les consommateurs marocain, le prix est l'un des supports de perception de l'image de marque. En effet, le consommateur prend en considération le prix de la voiture pour se construire une image de marque. Plusieurs études d'agences de communication ${ }^{6}$, ainsi que la notre, révèlent que les trois marques allemandes «Mercedes, Audi et BMW », ont les meilleures images

\footnotetext{
${ }^{5}$ Selon une étude de marché menée par FLANDERS INVESTMENT \& TRADE MARKET SURVEY, en 2015.

6 Dont l'agence de communication Euro RSCG C\&O. celle-ci a créé un outil de mesure de la valeur des marques, et a réalisé en 2010 une étude sur les marques automobiles auprès d'un échantillon de 1000 personnes.
} 
de marque. Leur seul déficit serait le rapport qualité/prix. Les marques devraient réussir à allier le prix à leur qualité. D'ailleurs plusieurs marques s'y sont lancées. A L'exemple de Mercedes qui propose aujourd'hui des modèles à des prix abordables, ainsi que la marque «Jaguar», qui a revu considérablement ses prix à la baisse.

\section{Apports de l'étude}

Nous considérons que cette étude détient un apport managérial très important. En effet, nous estimons avoir fourni aux managers des concessionnaires automobiles au niveau d'Agadir, un outil assez simple mais efficace, qui leur permettra de mieux positionner leur offre et surtout, la différencier par rapport aux concurrents, sur la base de l'image de marque.

\section{Limites de l'étude}

Il convient de préciser que notre travail présente certaines limites que nous tenons à évoquer. Il existe tout d'abord une limite liée à la validité externe de la recherche. Nos résultats sont obtenus sur la catégorie de produits automobiles, elles ne sont donc pas généralisables sur d'autres domaines. D'autre part, Notre enquête à porté sur un échantillon de convenance, plutôt que sur un échantillon représentatif de la population. De plus et pour des raisons de commodité, nous avons mené cette enquête uniquement au niveau d'Agadir, ce qui fait que les résultats peuvent être tout à fait différents dans d'autres villes, ou dans un pays différent.

\section{References:}

1. Aaker D. and Carman J. M. (1982). «Are you over-advertising?» Journal of Advertising Research 22.

2. Aaker D., Stayman D. M. and Hagerty M. R. (1986) «Warmth in Advertising: Measurement, Impact and Sequence Effects» Journal of Consumer Research, 12.

3. Aaker D.A. (1994) Le management du capital de marque, Dalloz.

4. Aaker J. (1997) «Dimensions of brand personality» Journal of Marketing Research: 347-356.

5. Abratt R. (1986) «Industrial buying in high-tech markets» Industrial marketing management, vol 15.

6. Aghekyan-Simonian M., Forsythe S., Suk Kwon W. and Chattaraman, V. (2012) «The role of product brand image and online store image on perceived risks and online purchase intentions for apparel. » Journal of Retailing and Consumer Services, 19(3).

7. Balmer, J. (2008) «Identity based views of the corporation: Insights from corporate identity, organisational identity, social identity, visual 
identity, corporate brand identity and corporate image» European Journal of Marketing, 42.

8. Batra R., Lehman D.R, Burke J., Pae. J. (1995) «When does advertising have an impact, A Study of Tracking Data», Journal of Advertising Research, 35.

9. Bloemer J., Ruyter K. and Peeters P. (1998) «Investigating drivers of bank loyalty: The comple relationship between image, service quality and satisfaction» The International Journal of Bank Marketing, 16.

10. Cian, L. (2011) «How to measure brand image: a reasoned review. » The Marketing Review, 11.

11. Dodds W., Monroe K., and Grewal, D. (1991) «Effects of Price, Brand, and Store Information on Buyers' Product Evaluations». Journal of Marketing Research, 28.

12. Erickson G. M., and Johansson J. K. (1985) «The role of price in multiattribute product evaluations» Journal of Consumer Research, 12.

13. Evrard Y., Pras B., Roux E. (2000), Market, Dunod, Paris.

14. Holbrook, M., and Batra, R. (1987). «Assessing the Role of Emotions as Mediators of Consumer Responses to Advertising» Journal of Consumer Research, 14.

15. Hu H. and Jasper C. (2006) «Social cues in the store environment and their impact on store image» International Journal of Retail \& Distribution Management, 34.

16. Huang W (2010) «Brand Story and Perceived Brand Image: Evidence from Taiwan. » Journal of Family and Economic Issues. 31.

17. Keller KL. (1993) «Conceptualizing, measuring, and managing customer-based brand equity» Journal of Marketing 57.

18. Keller, K.L. (2009) «Building strong brands in a modern marketing communications environment» Journal of Marketing Communications, 15.

19. Keller, K.L. (2009) «Building strong brands in a modern marketing communications environment» Journal of Marketing Communications, 15.

20. Kim J.H. et Hyun Y.J., (2011) «A model to investigate the influence of marketing-mix efforts and corporate image on brand equity in the IT software sector» Industrial Marketing Management, Vol 40.

21. Morisse B. (2004) Le processus de valorisation de l'offre dans le comportement d'achat : une application au cas automobile, Thèse de doctorat en sciences de gestion,, Université de Caen / BasseNormandie.

22. Qanqom A. (2018). «La Perception De L'image De Marque Automobile: Étude Qualitative Exploratoire Auprès Des 
Consommateurs De La Ville d'Agadir » European Scientific Journal, Vol $14 \mathrm{~N} 25$.

23. Rao A., and Monroe K. (1989). «The Effect of Price, Brand Name, and Store Name on Buyers' Perceptions of Product Quality: An Integrative Review» Journal of Marketing Research, 26.

24. Ratier M. (2006) La perception de l'image d'une marque automobile par le consommateur : proposition d'un modèle, Thèse de doctorat en sciences de gestion, Toulouse 1.

25. Salciuviene L., Lee K. and Yu C. (2007) «The impact of brand image dimensions on brand preference» Economics and Management, 12(1).

26. Schmitt B. (2012) «The consumer psychology of brands» Journal of Consumer Psychology, 22.

27. Sharma A., Krishnan R. and Grewal D. (2001), «Value Creation in Markets. A Critical Area of Focus for Business-to-Business Markets» Industrial Marketing Management.

28. Simon C. J., and Sullivan M. W. (1993) «The Measurement and Determinants of Brand Equity: A Financial Approach. » Marketing Science 12.

29. Soulez S. (2005) Le comportement d'achat des clients professionnels : un espace entre $B$ to $B$ et $B$ to $C$, Thèse de doctorat en sciences de gestion, Université Paris I - Panthéon Sorbonne.

30. Sproles, E. K., Sproles G. B. (1990) «Consumer decision-making styles as a function of individual learning styles» Journal of Consumers Affairs. 24(1).

31. Van Riel A.C.R., De Mortanges C.P. and Streukens S. (2005), «Marketing Antecedents of Industrial Brand Equity: An Empirical Investigation in Specialty Chemicals», Industrial Marketing Management, 34.

32. Voelckner, F., and Sattler, H. (2005) « Empirical generalizability of consumer evaluations of brand extensions. » Research paper No. 25.

33. Winchester M.K. and Fletcher M. (2000) «Calibrating your brand image measurement technique by utilizing empirical generalizations» The Journal of Brand Management.

34. Yoo B., Donthu N. (2001) «Developing and validating a multidimensional consumer-based brand equity scale» Journal of Business Research 52.

35. Yoon E, Guffey H.J. and Kijewski V. (1993), «The Effect of Information and Company Reputation on Intentions to buy a Business Service», Journal of Business Research, $\mathrm{n}^{\circ} 27$.

36. Zinkhan G. M. and Burton S. (1989) «Refining a Multidimensional Profile For Television Commercials: an Application of Targer Analysis», Advances in Consumer Research, Volume 16. 